\title{
ARTÍCULO \\ ANALISIS ANTROPOLÓGICO DE LAS RELACIONES HUMANAS COMO REFERENTE DEL CAMBIO ORGANIZACIONAL
}

\author{
Rocío Abigail Tinajero García \\ Centro Universitario Oparin \\ abi.tinajerogarcia@gmail.com
}

DOI: https://doi.org/10.38128/cienciayfilosofa.v3i3.17

\section{RESUMEN}

Se presenta una propuesta de Modelo de Análisis del Sistema Organizacional para el individuo encargado del talento humano en las organizaciones, a fin de delimitar los factores culturales, sociales y de adaptación que influyen para un cambio organizacional.

PALABRAS CLAVES: Organización, Pública, Privada, Antropología, Culturación.

\section{ASBTRACT}

A proposal for an Organizational System Analysis Model is presented for the individual in charge of human talent in organizations, in order to define the cultural, social and adaptation factors that influence organizational change.

KEY WORDS: Organization, Public, Private, Anthropology, Culture.

RECIBIDO: 23.5: 2020

APROBADO: 05.06:2020

Ciencia y Filosofía | Revista de Ciencias Sociales y Humanidades, vol. 3, núm. 3, ANALISIS ANTROPOLóGICo DE LAS RELACIONES HUMANAS COMO REFERENTE DEL CAMBIO ORGANIZACIONAL por Rocío Abigail Tinajero García, pp. 32-49 


\section{INTRODUCCIÓN}

Las organizaciones son estructuras sociales donde se desarrollan situaciones laborales, profesionales, económicas y sobre todo relaciones humanas. Así, para Susan Wright (2005) propone que por cultura organizacional se debe entender, de acuerdo a la concepción semiótica de cultura sostenida, un sistema de redes de significado a partir de las cuales los actores interpretan sus propias prácticas negociando significados" (Gonnet, 2012: 9)

Sin embargo, también se advierte sobre la pluralidad de significados que describen el fenómeno de la organización empresarial, que van desde la analogía biológica del ADN de las empresas, pasando por la analogía de la vertebración, es decir como columna vertebral; también como rectoría en la cual se fincan todos los valores, las políticas, las estrategias hasta la visión antropológica que considera que el factor humano, las relaciones de trabajo son constituyentes para comprender valores y costumbres, así como su interacción en el entorno social, familiar y cultural en cuanto objeto de estudio.

La investigación que se propone aquí, toma en cuenta la diversidad cultural en un contexto globalizado y cambiante en donde se comprende lo administrativo con una tendencia a controlar las acciones internas y externas de las organizaciones, generando contextos culturales, adaptativos y multifuncionales en las relaciones humanas derivadas de manera histórica, es decir emerge un paradigma organizacional de corte mecanicista de control social en el que se asumía sin reparos que los flujos de capital creaban valorización que ofrecía ventajas sobre el control externo logrado a través de premios y sanciones. (Pfeffer, 2000: 168).

De lo anterior, se deriva la propuesta de un modelo de culturación entendido como medio para reivindicar la figura social organizativa, plasmada como medio social para 
forjar una identidad objetiva del factor empresa, que pueda observarse tanto desde un punto de vista pluricultural como desde una postura sociológica.

No obstante, se advierte que no se trata de un pleonasmo en el sentido de utilizar el término "cultura" para indicar una "cultura en el factor empresarial" pues esto invita al discurso esencialista para definir el fenómeno de la organización. Por el contrario, desde un enfoque filosófico-antropológico, se analizan los principales problemas adaptativos de las organizaciones que radican en las diferencias culturales, formando barreras para generar cambios significativos, cuantitativos y susceptibles de valorizarse, es decir poder ponderar múltiples situaciones reflejadas en valores estadísticos, los cuales, con el tiempo, son susceptibles de cambio.

Conforme al compromiso epistémico propuesto por Bachelard (2009: 15) en el cual: una experiencia nueva le dice no a la experiencia anterior, es decir el modelo de culturación le dice no al modelo mecanicista de control social basado en premios y casigos.

Para ello, se ha dividido la investigación en cuatro apartados, el primero denominado la Brujula moral en el que se aborda cómo se construye la Cultura Organizacional y la similitud de ésta con la forma en cómo se define la Cultura del individuo, es decir se demuestra la simbiosis que existe entre ambas culturas.

Un Segundo momento está dedicado al Desarrollo Organizaciones (DO) a través del cual, las organizaciones evalúan al individuo en relación a su desempeño y sus conductas dentro de la organización a través de la valoración y gestión de resultados y evaluación de competencias que permite a las organizaciones identificar el talento y el potencial de los miembros que la integran, generando una razón cuantitativa que respalda la importancia de la simbiosis de la cultura individual y la cultura de la empresa.

En el tercer apartado, se refiere a cómo la evaluación es la base para identificar a los individuos de alto potencial en la organización donde los factores Social-Antropológico es la base para crear éxito dentro de una organización, es decir que estos factores son los que gestan la aspiración y el compromiso, para convertirse en colaboradores de alto potencial . 
Por último, se examinará la categoría de liderazgo entendido como pilar fundamental esperado para los colaboradores de alto potencial, se habla de este rol en su responsabilidad de promotor del cambio y del cómo, el liderazgo ejecutado en un estilo transformacional es pieza clave para un cambio cultural en las empresas.

\section{I.LA BRÚJULA MORAL}

Una de las formas más efectivas y simples de mirar, conocer y analizar las organizaciones dentro del ámbito empresarial, es sin duda haciendo un comparativo de estas, con la más antigua de todas las estructuras: la familia.

Ambas estructuras tienen, formal o informalmente, una cabeza (líder), unos miembros (equipo) y objetivos comunes que perseguir (objetivos).

Figura 1: Organización familiar (tradicional en verde) y empresarial (en azul).

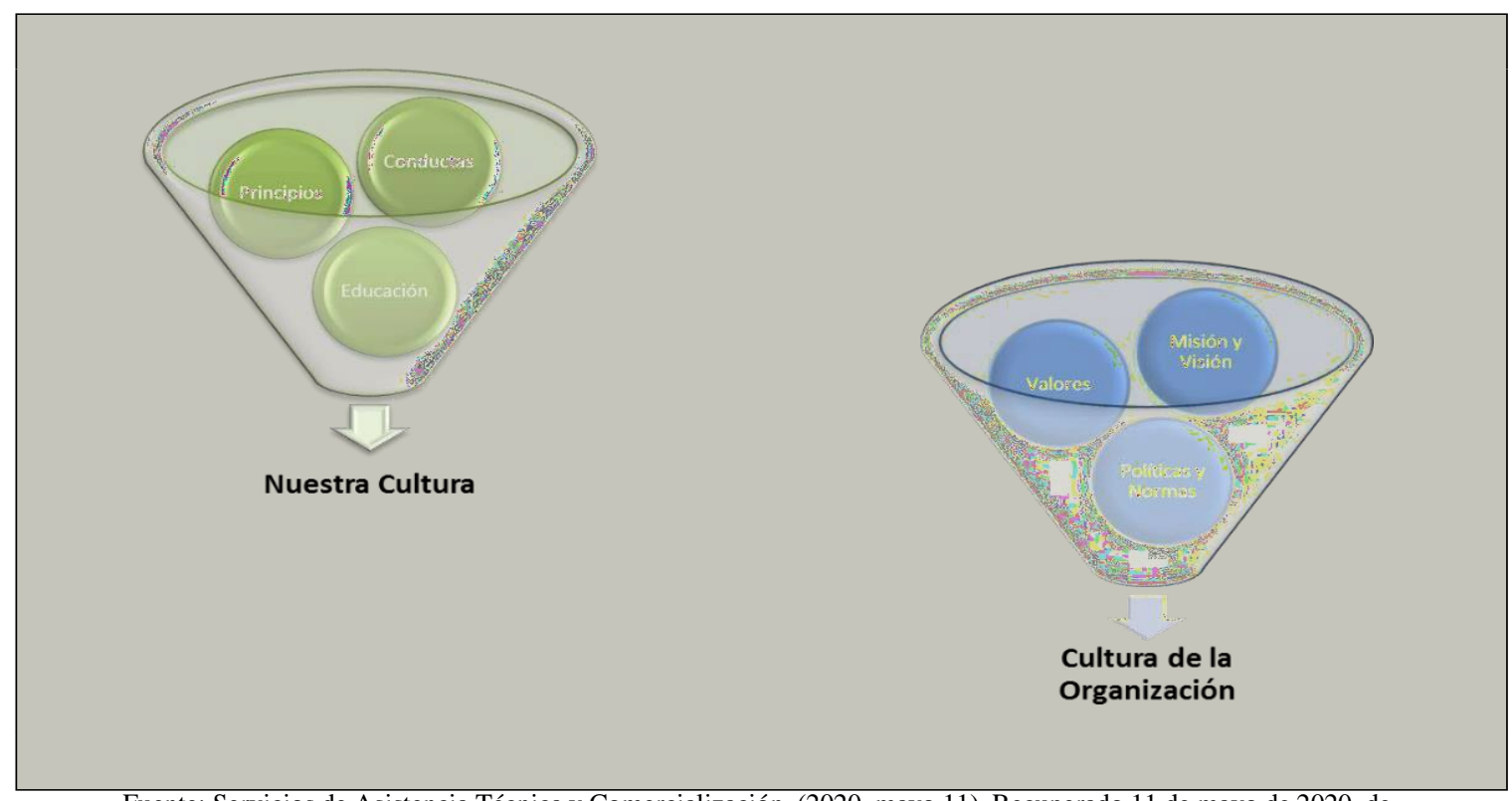

Fuente: Servicios de Asistencia Técnica y Comercialización. (2020, mayo 11). Recuperado 11 de mayo de 2020, de https://www.linkedin.com/pulse/la-diferencia-entre-talento-y-potencial-s-c-/ 
El trabajo del entorno se obtiene se considera bien colectivo, cuya motivación podrá ser por "convencimiento" o por "vencimiento" enmarcados en una mezcla de principios, valores y normativas que regulen su actuar, y en tanto ese actuar no esté basado en el auto convencimiento o, por lo menos, en la autorregulación, no podemos decir que haya presencia de algún tipo de evolución en la organización.

A sí como en el núcleo familiar existen roles (papas, hijos y abuelos) y objetivos en común (sustento, alimento, vestido), todos ellos "trabajando" y siendo "evaluados" en un entorno "controlado" a través de las reglas de familia y de su entorno social; Análogamente en las organizaciones aparecen los mismos "personajes" actuados por un jefe y un equipo de colaboradores diferentes entre sí, buscando alcanzar las metas establecidas por el negocio, actuando, siendo evaluados y teniendo como base la denominada "Cultura Organizacional", que no son más que los valores éticos, competencias o conductas esperadas, las reglas y normativas empresariales, como se muestra a continuación:

Figura 2: Cultura Organizacional dentro de una empresa

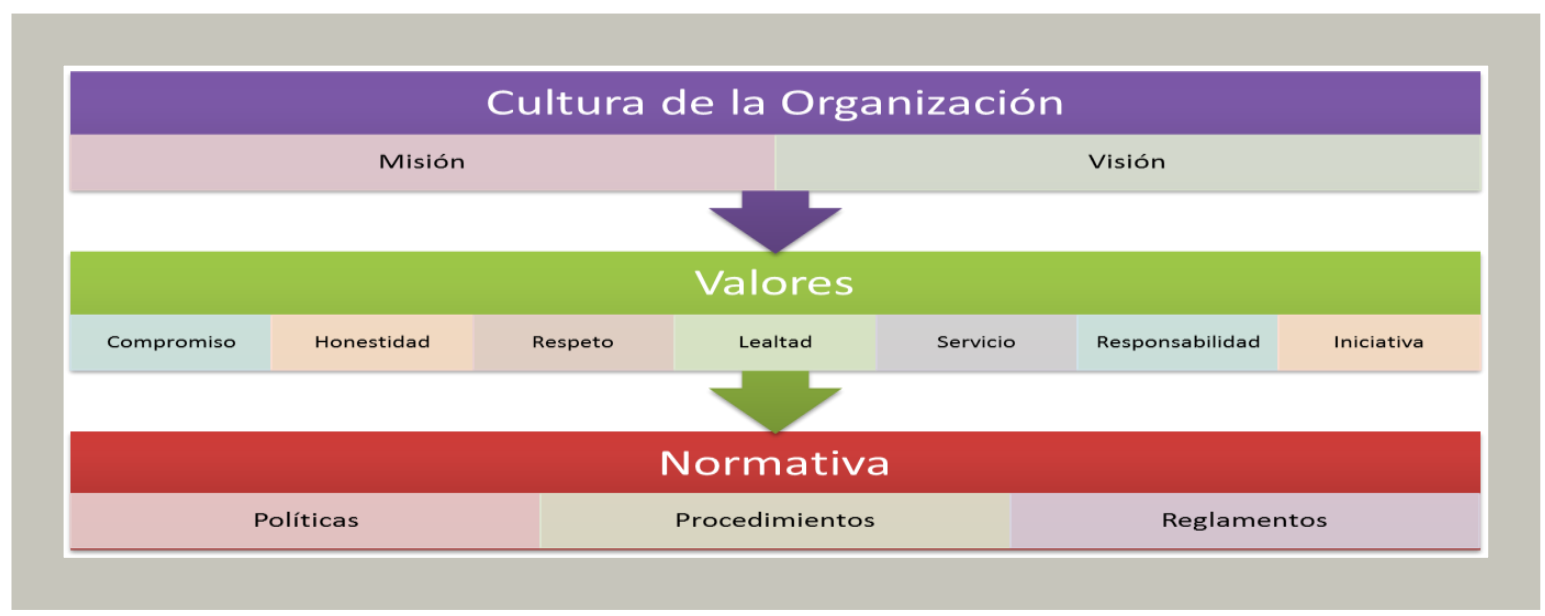

Fuente. Servicios de Asistencia Técnica y Comercialización. (2020, mayo 11). Recuperado 11 de mayo de 2020, de https://www.linkedin.com/pulse/la-diferencia-entre-talento-y-potencial-s-c-/

Ciencia y Filosofía | Revista de Ciencias Sociales y Humanidades, vol. 3, núm. 3, ANALISIS ANTROPOLÓGICO DE LAS RELACIONES HUMANAS COMO REFERENTE DEL CAMBIO ORGANIZACIONAL por Rocío Abigail Tinajero García, pp. 32-49 
Para lograr la evolución referida, existen diferentes metodologías, las cuales en su conjunto componen el Desarrollo Organizacional.

El Desarrollo Organizacional (DO) se compone de metodologías y herramientas que permiten a las organizaciones entrar en un proceso evolutivo a través del establecimiento de roles, metas, indicadores de desempeño y métricas, que después de un proceso de evaluación de mediano plazo, permiten conocer los niveles de desempeño de la Organización, mapear el talento dentro de la misma e identificar aquellos miembros con potencial para el sostenimiento del negocio.

\section{EL DESARROLLO ORGANIZACIONAL}

Para entender el DO, se debe considerar primero la existencia de cuatro aspectos que lo componen, y aunque a simple vista parecieran ser lo mismo, la tenue diferencia entre ellos tiene una gran importancia cuando la evolución de las organizaciones se refiere:

i. Los Roles.- Constituidos por las descripciones de los cargos de cada miembro de la Organización, en donde se detallan las principales funciones que soportan la razón de ser de sus cargos y su impacto y criticidad dentro de la organización, teniendo la visual de que un cargo critico es solo aquel que tiene un rol supervisorio.

ii. La Medición del Desempeño.- Conformado por los indicadores, las metas, las métricas y los periodos de evaluación de cada uno de los cargos de la organización, los cuales serán evaluados en su cumplimiento a través de diferentes herramientas, siendo las más conocida la Talent Management (9 o 12 casillas).

iii. El Portafolio de Talento.- Es el mapa dentro del cual se posicionan cada uno de los miembros de una organización, considerando sus resultados de desempeño (el Qué) y la evaluación de sus competencias (el Cómo). Estas últimas se derivan de la Cultura Organizacional y se pueden evaluar aplicando una simple fórmula:

\section{Comportamiento (lo que se espera que el colaborador haga)}

Ciencia y Filosofía I Revista de Ciencias Sociales y Humanidades, vol. 3, núm. 3, ANALISIS ANTROPOLÓGICO DE LAS RELACIONES HUMANAS COMO REFERENTE DEL CAMBIO ORGANIZACIONAL por Rocío Abigail Tinajero García, pp. 32-4 


\section{$X$}

Frecuencia Observada (lo hace casi nunca, a veces, frecuentemente o casi siempre)

\section{$=$ Competencia}

La evaluación popuesta a quí, puede ser obtenida con varios niveles de profundidad, dependiendo el interés de la empresa y el tamaño de la estructura organizacional, lo cual se suguiere esquema organizacional:

- $90^{\circ}$ (supervisor - colaborador);

- $180^{\circ}$ (supervisor - colaborador - jefe del supervisor);

- $270^{\circ}$ (supervisor - colaborador, jefe del supervisor - pares del colaborador);

- $360^{\circ}$ (supervisor - colaborador - jefe del supervisor - pares del colaborador y el equipo del colaborador).

Etimologicamente potencial se deriva del latín, proviene del vocablo potis que significa "poder", el nexo - $n t_{-}$- que equivale a "agente", y el sufijo - $a l$ - que puede traducirse como "relativo a". Es decir, "el poder relativo a la gente".

En términos organizaciones Potencial del Talento, de acuerdo a Todaro y Smith (2015: 16) indican que el capital humano se define como las inversiones productivas hechas en las personas, como habilidades, capacidades, competencias y salud resultado del gasto en educación, en programas de entrenamiento dentro del trabajo y atención médica, que se traduce en el ámbito empresarial como generaciones del Talento, es decir Organizaciones de colaboradores con Potencial, que aseguren el sostenimiento del negocio en el tiempo.

Sin embargo, se advierte que existen diferentes definiciones de Talento y Potencial. Al respecto, Scullion, Collings \& Caligiuri (2010: 106) sostienen que "todas las actividades que la organización realiza con el fin de atraer, seleccionar, desarrollar y retener a los mejores empleados en los roles más estratégicos (los roles necesarios para alcanzar 
las prioridades estratégicas de la organización) a escala mundial". Si bien cada vez hay más empresas que cuentan con sistemas mundiales para identificar y desarrollar el talento, una parte considerable de los mismos se describen como adopción de un "enfoque ad hoc o sin ningún orden establecido" (Mc Donnell et al., 2010: 150)

En un contexto organizacional en donde prevalence la autoreferencia (Finlay, 2012: 24) El talento se transforma en una manifestación de la inteligencia emocional en cuanto aptitud o conjunto de aptitudes o destrezas sobresalientes respecto de un grupo para realizar una tarea determinada en forma exitosa.

Para Arraiz (2002: 135-136) «[...] el potencial es la capacidad de las personas de adaptarse y desempeñar a satisfacción puestos de superior nivel de responsabilidad del que desempeñan en un momento dado. Una persona con alto potencial es una persona promocionable». de mayor nivel en la Organización».

Mientras que para Pereda y Berocal (2006: 255), el potencial se refiere al futuro del trabajador, «[...] es lo que la persona puede llegar a hacer o a ser dentro de la organización». Dina Sznirer define el potencial como «[...] la capacidad actual o proyectada de un individuo para operar exitosamente en el futuro en puestos de mayor jerarquía o complejidad»

Portanto, se puede decir que exsite una diferencia entre potencial y talent ya que talento, está más asociado con el "saber hacer" y "poder hacer", el mientras que potencial se enfoca principalmente en el "querer hacer"; es decir, el potencial se convierte en el principal detonador de la acción, donde influyen variables como la voluntad, el deseo y la búsqueda de un beneficio. De allí el adagio popular que dice: si no sabes, te enseño; si no puedes, te ayudo; pero si no quieres, no hay manera de hacer nada.

En sentido hipotético, una empresa de Consultoría Servicios de Asistencia Técnica y Comercialización, S. C., en donde el objetivo fue el diseño e implementación de un Modelo de Gestión de Alto Desempeño, para evaluar a todos los empleados administrativos.

Después de analizar los factores que se describen a continuación: 
Figura 3 denominada Pirámide Conductual

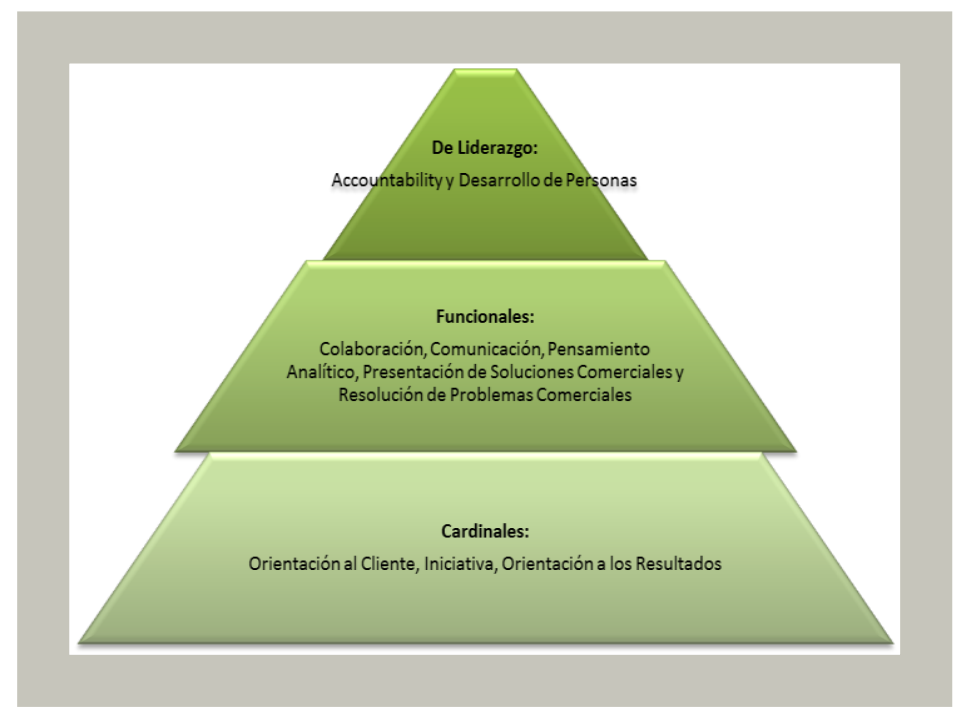

Fuente:Servicios de Asistencia Técnica y Comercialización. (2020, mayo 11). Recuperado 11 de mayo de 2020, de https://www.linkedin.com/pulse/la-diferencia-entre-talento-y-potencial-s-c-/

Competencias Cardinales.- Aquellas exigidas para todos los niveles de la organización, sin importar su nivel de aporte.

Competencias Funcionales.- Las directamente ligadas al nivel de contribución al negocio y responsabilidades del cargo / área funcional (ventas, recursos humanos, finanzas, manufactura, etc.).

Competencias de Liderazgo.- Evaluables exclusivamente para las funciones que tienen equipos a cargo.

Posteriormente, una vez obtenida los resultados de los indicadores de gestión de todos y cada uno de los colaboradores, en un rango de evaluación de 12 meses, se determinó un tablero de talento de 9 casillas (ver capitulo II) para obtener el Portafolio de Talento de la Organización. 


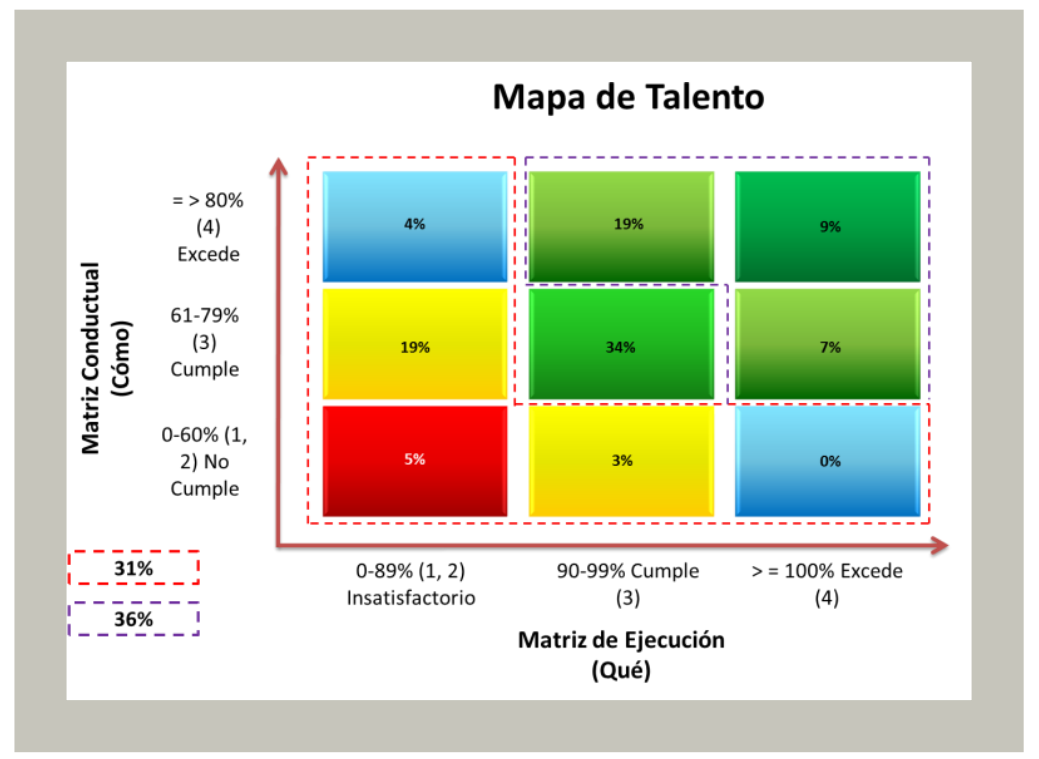

Fuente: Servicios de Asistencia Técnica y Comercialización. (2020, mayo 11). Recuperado 11 de mayo de 2020, de https://www.linkedin.com/pulse/la-diferencia-entre-talento-y-potencial-s-c-/

En este ejercicio se observa que la distribución de talento, después de evaluación de $90^{\circ}$, fue de un $31 \%$ de colaborador por debajo del desempeño promedio esperado, $34 \%$ con un desempeño dentro del promedio y un $36 \%$ de colaboradores que se podrían considerar como talentosos o de alto desempeño. Como referencia, y de acuerdo a la curva de distribución normal de este tipo de estudios, los porcentajes son $15 \%, 65 \%$ y $20 \%$, respectivamente.

El talento tiene como base de ejecución, por supuesto el Talento; sin embargo, si la persona no tiene motivadores que lo lleven a la acción, el Talento no sirve de nada. A través de diferentes estudios en el marco de las organizaciones, estas variables que impulsan a la acción se han concentrado en dos: El Compromiso y la Aspiración.

Cuando combinamos estas dos variables con el Talento (Habilidades), hemos completado el círculo virtuoso del saber, el poder y el querer hacer, lo que en su conjunto, y refiriéndose al ámbito empresarial, se le conoce como Potencial. 
Sin embargo, existen diferentes niveles de Potencial que están definidos en función de la combinación de estas variables. De lo anterior, tenemos la siguiente imagen:

Figura 4.- Diagrama de alto potencial.

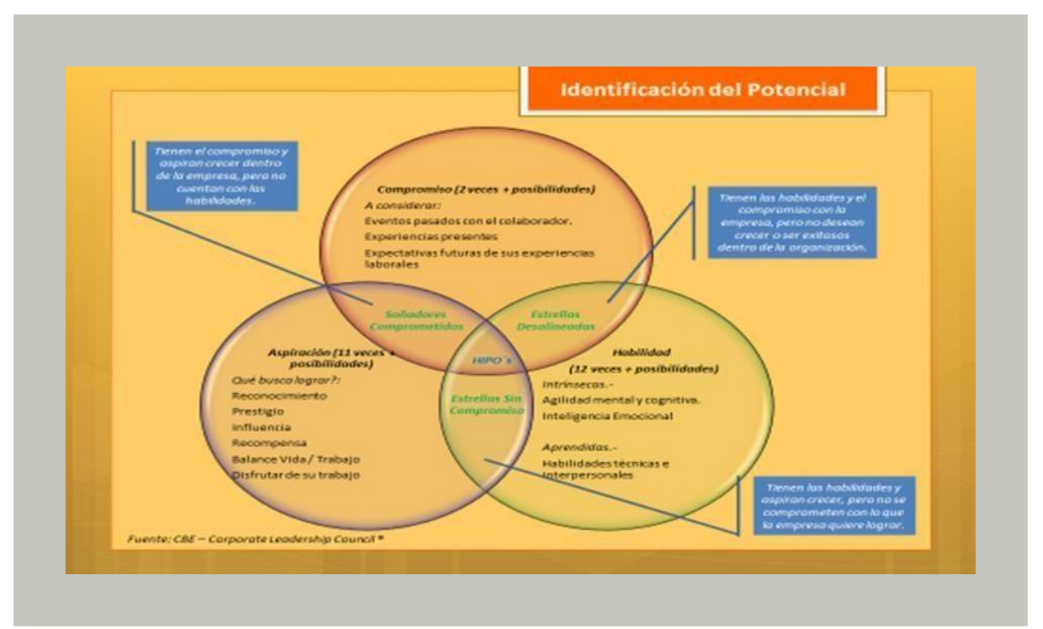

Fuente: CORPORATE LEADER SHIP COUNCIL. (2015, julio 16). Cracking the code on high potential finding engaging and retaining your best webina. Recuperado 7 de mayo de 2020, de https://www.slideshare.net/humancapitalmedia/cracking-the-codeon-high-potential-finding-engaging-and-retaining-your-best-webinar?from_action=save

En este diagrama se ubican los diferentes niveles de Potencial, refiriéndose a los Altos Potenciales (HIPO's, por sus siglas en inglés - High Potencial) como aquellos que combinan las tres variables; sin embargo, cuando alguna de las variables no está contenida dentro de la persona, se observan distractores que evitan ese alto potencial.

Por ejemplo, aquellas personas que cuentan con el talento (habilidades) y aspiran a desarrollarse dentro de la organización, pero carecen de un compromiso con lo que la empresa quiere lograr; es decir, no se han apropiado de la Misión y la Visión del negocio, son definidas sin identidad y compromiso con la organización.

Estas personas "pueden" y "quieren" tener un desarrollo personal, pero en definitiva no "pretenden" alcanzarlo dentro de la organización en la cual se encuentran; toda vez que el objetivo planteado por la misma (Misión y Visión), no es suficiente para generarle un compromiso. 


\section{EL D.O. COMO DETONANTE DEL CAMBIO ORGANIZACIONAL}

De igual modo, se tuvo en cuenta la percepción de Felipe Cáceres-Rubio y Mónica VillacrésChaparro (2010), sobre cultura organizacional, debido a que surge de la agrupación de seres humanos, que persigue una finalidad específica, e implica una organización sociocultural, en la que se establecen normas y valores que orienten la conducta colectiva y ahí, los individuos tejen una serie de imaginarios sociales. Cuando las organizaciones han definido claramente la estrategia y metodología a seguir para medir el desempeño de sus colaboradores, buscando asegurar que los logros individuales se alineen y sumen para el logro colectivo, es decir, que la "sucesión de puntos nos den una línea recta”, el reto principal ahora radica en lograr conjuntar ese talento con la aspiración y el compromiso, para obtener una estructura organizacional de alto potencial.

Para lograr lo anterior, se debe lograr que esa estrategia y metodología utilizada se convierta para medir el talento se convierta en una Cultura. Una Cultura en la cual no solo se transmita lo que se debe hacer, sino que se logre un nivel de apropiación tal que la organización quede convencida de que ese es el deber ser.

Para ello, el ejercicio de congruencia en las acciones y la coherencia de mando se vuelven factores fundamentales para lograr credibilidad con este nueva Cultura, en donde los líderes de la Organización son pieza clave para el éxito.

Los líderes deben ser modelo a seguir y los principales agentes de cambio, predicando con el ejemplo y reconociendo logros y fracasos para posicionarse con personas a los que el resto de la Organización quieran seguir.

Estos líderes deberán demostrar conductas esenciales que van más allá de los estilos de liderazgo tradicionales (tutorial, coercitivo, marcapasos, democrático y directivo), a través de las cuales logren, en conjunto con factores como la continuidad de los procesos, la comunicación efectiva y el reconocimiento objetivo, convertir un Modelo de Desarrollo Organizacional en una Cultura Organizacional viviente y palpable para el resto de la Organización.

Ciencia y Filosofía | Revista de Ciencias Sociales y Humanidades, vol. 3, núm. 3, ANALISIS ANTROPOLógICo DE LAS RELACIONES HUMANAS COMO REFERENTE DEL CAMBIO ORGANIZACIONAL por Rocío Abigail Tinajero García, pp. 32-49 
Anteriormente hablábamos de la importancia de hacer las cosas por convencimiento, más que por vencimiento, y la razón de ello es que para lograr que los procesos, metodologías y acciones se implementen de manera cotidiana evolucionenhasta convertirse en parte de la Cultura organizacional, debemos lograr un profundo convencimiento de esto en cada uno de los colaboradores. Esto significa que se ha definido a la cultura como una "variable" que afecta externa o internamente a la organización, en un sentido más o menos positivo o negativo para sus dos finalidades: el sistema de gestión y control organizacional (Gonnet, 2012, p. 8).

El proceso de implementación de Cultura puede visualizarse en tres momentos. El primero corresponde a la etapa en donde la administración de la Cultura se realiza sin más apoyo que los usos y costumbres, en donde las relaciones humanas entre líderes y estructura están totalmente basadas en la idiosincrasia de una o pocas personas; lo cual es característico de las empresas familiares, por ejemplo.

El segundo momento es la etapa en donde la Cultura ha tenido una evolución al reconocer que esos usos y costumbres deben ser formalizados en Políticas y Procedimientos, para dejar de lado la imagen de que "en la empresa se hace lo que el jefe dice", para ahora disfrazar el hecho con el establecimiento de una "Normativa".

La tercera y última etapa ya implica un proceso no solamente evolutivo, sino de madurez organizacional, en donde los líderes reconocer que la mejor forma de "administrar" la Cultura es a través del convencimiento de cada uno de los miembros que componen la estructura organizacional de la empresa, quien se autorregularán en su actuar porque están convencidos que así debe ser en beneficio propio y de la empresa, y porque exista un medio coercitivo que les imponga un "castigo" por no conducirse en el deber ser.

En la figura siguiente se observan las diferentes etapas, así como los niveles de impacto y permanencia de la Cultura en cada una de ellas. 


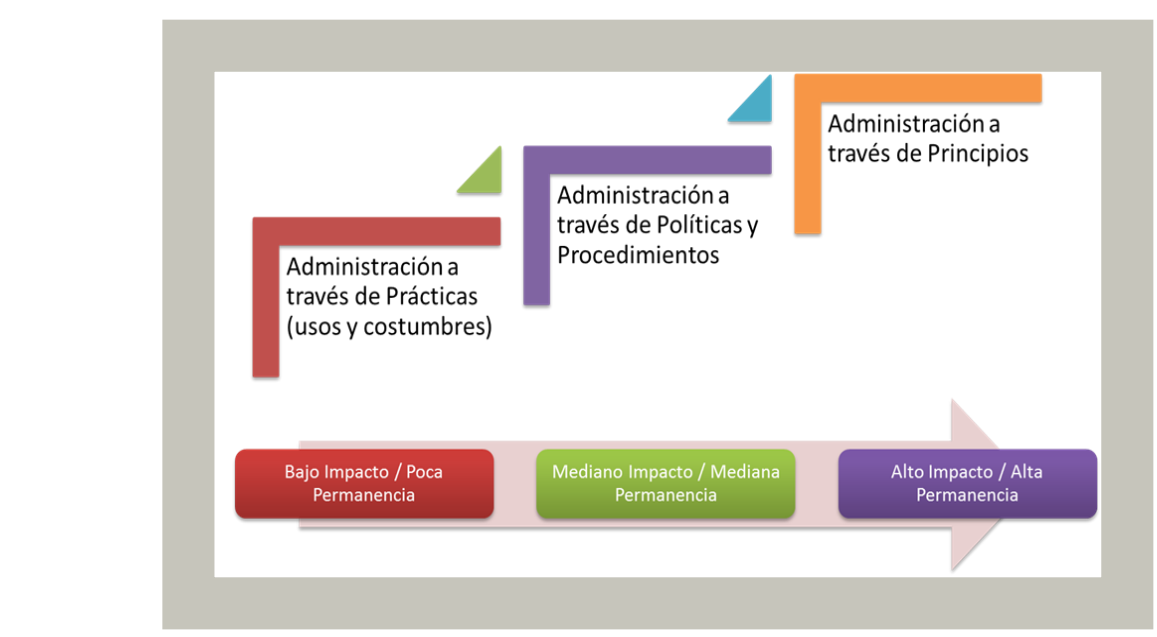

Fuente: Servicios de Asistencia Técnica y Comercialización. (2020, mayo 11). Recuperado 11 de mayo de 2020, de https://www.linkedin.com/pulse/la-diferencia-entre-talento-y-potencial-s-c-/

De lo anterior, la importancia de que las relaciones humanas se basen en aspectos como la confianza, la coherencia y el respeto, con lo cual se alcanzará una organización en donde exista una fuerte alineación entre los principios y valores como empresa y los principios y valores del individuo; logrando con ello que el Talento se combine con la Aspiración, con un alto Compromiso, para que todo gire en torno a una Estructura Organizacional de Alto Potencial.

\section{IV.EL LÍDER COMO AGENTE DE CAMBIO}

La principal responsabilidad de un líder es, sin duda, lograr los objetivos a través de otros. Para ello, un líder debe contar con ciertas competencias que no necesariamente pueden ser adquiridas a través de Programas de Desarrollo; es más, se podría decir que la mayoría de estas ya están contenidas en el ADN del individuo. Forman parte de su historia, principios, valores, principalmente, de su forma de ver las situaciones de la vida y a la humidad como tal. 
La demostración frecuente de estas competencias lo ayudará a construir paulatinamente su imagen, su coherencia de mando y su congruencia en el actuar, lo cual será pieza clave para ejercer un liderazgo transformacional dentro de la organización. Este liderazgo transformacional, a su vez, lo posicionará como un aliado estratégico en la toma de decisiones de empresa, promoviendo y acompañando a sus líderes y pares hacia el éxito de mediano y largo plazo.

"El liderazgo es el arte de conseguir que otra persona haga algo que quieres hacer, porque quiere hacerlo." (Einsenhower, Dwight. Presidente de EUA (1953 - 1961)), y en esencia así es. Para lograrlo, fue necesario que las de un líder sufrieran una evolución, principalmente tres de ellas.

Compasión. - Resultado de la evolución de una de las competencias clásicas requeridas por el área de Recursos Humanos, denomina Empatía, la Compasión va más allá del simple hecho de "ponerse los zapatos del otro".

Significa involucrarse en la persona más allá del hecho de una relación Jefe / Colaborador, conocer sus éxitos pasados, preferencias, aspiraciones y habilidades, pero también sus miedos, fracasos, limitaciones y disgustos; y entender que somos diferentes entre sí y que la equidad a veces debe estar en vez de la igualdad.

Humildad. - La cual es sin duda una de las competencias más complicadas de desarrollar, toda vez que es contrariamente alimentada conforme el líder crece y se posiciona gracias a sus resultados.

Implica un alto nivel de objetividad, apertura, escucha activa y, principalmente, un proceso constante de aprendizaje y desaprendizaje, a través de lo cual el líder asimila que no tiene la verdad absoluta, no tiene el conocimiento completo ni la solución a todos los problemas, pero lo más importante, es que entiende y acepta que no hay nada de malo en ello; siempre y cuando se rodee de personas que tienen, y hacen bien, aquello de lo que él carece. 
Sentido de Logro. - Pero no desde un punto de vista personal, egoísta y ególatra, sino a través del uso de las dos competencias anteriores, que producen en el líder un sentimiento de gusto por los éxitos de los demás.

Implica estar dispuesto a ceder su lugar bajo el reflector, en aras de lograr que las cosas sucedan. Tener claro que en las empresas existe una organización formal y una informal, así como "líderes de opinión”, a los cuales puede y debe pedir apoyo para lograr el bien colectivo, sin importar quien se "cuelgue la medalla".

A través de estas competencias, combinadas con su imagen, coherencia de mando y congruencia en el actuar, el líder conseguirá que su equipo y el resto de la Organización no solo dispongan de un destino y una brújula para avanzar, sino que además encuentren y sientan como propio el motivo para hacer el viaje, de la manera tal que harán la travesía, aun sin estar líder presente. 


\section{CONCLUSIONES}

Las empresas que busquen mejorar la filosofía de la organización deberán gestar certidumbre en el entorno socio cultural del factor humano para lograr el correcto desarrollo organizacional y lograr un equilibrio entre misión, visión y valores, fusionando el factor humano con la economía del entorno socio cultural que fecunde pensamientos enfocados al desarrollo y nivel de competitividad de éste.

Las organizaciones que logren germinar la semilla del desarrollo antropológico en cuanto factor humano para dar paso a la interculturalidad organizacional logrando la aceptación e identificación del individuo como Factor determinante para el cambio de la cultura institucional.

Queda demostrado que utilizando liderazgo, trabajo colaborativo, alcance de objetivos, metas y cambios estratégicos con miras al perfeccionamiento de la organización, declinando con ello el paradigma de la motivación por premios y castigos practicada durante mucho tiempo en un estadio de desarrollo posindustrial.

Por el contrario la mudanza que suscita el tipo de organización conectada en diferentes formatos tecnológicos motiva las competencias que los individuos desarrollana lo largo del desarrollo intercultural, el talento y potencial para desempeñar las funciones, así como el compromiso que esto detona al interiorizar actitudes y compromiso del factor humano para cumplir las políticas organizacionales.

La Gestión del Talento (GT) encaminado al Cambio Organizacional es prueba del impacto obtenido, es decir fusionando talento, potencial, actitudes, valores, trabajo en equipo, para logar el impacto de Referente del Cambio Organizacional. 


\section{REFERENCIAS}

BACHELARD, G (2009). La filosofía del no, Buenos Aires: Amorroutu.

FINLAY, A (2012). Cómo comunicar la investigación para influir: estrategias y desafíos para generar el cambio, Sudáfrica: APC.

TODARO Y SMITH, M (2015). Economic Development, Massachuset: Pearson.

\section{REVISTAS.}

CÁCERES-RUBIO, et al (2010). “Cultura organizacional y las fusiones empresariales”. En Revista Universidad de La Sabana, vol. pp.

COVEY, S (1998). El Liderazgo Centrado en Principios, México: Paidos.

GONNET, J (2012). “Cultura, organizaciones y antropología”. Revista de Antropología, vol. 21, pp. 1-20.

ORANTOS, A (2016). "La excelencia y la sobrevaloración del talento”, España: Fh

SCULLION, H, et al (2010). "Global talent management", Journal of World Business, Vol.45 No.2, pp. 105-108. 